\title{
NATURAL INTERACTION OF GAME-BASED LEARNING FOR ELASTICITY
}

\author{
Maryam Savari ${ }^{1}$, Mohamad Nizam Bin Ayub ${ }^{2}$, Ainuddin Wahid Bin Abdul Wahab ${ }^{3}$, Nurul Fazmidar Mohd. \\ Noor $^{4}$ \\ 1,2,3,4 Faculty of Computer Science and Information Technology \\ University of Malaya \\ 50603 Kuala Lumpur \\ Malaysia
}

Email: maryamsavari@gmail.com¹,nizam_ayub@um.edu.my²

\begin{abstract}
Game-based Learning (GBL) is an alternative method for learners to experiencing a volatile environment in a safe place. In this environment, an alternative technique is required to facilitate the learning and to prevent injuries and potential hazards. Subjects involving elasticity are commonly considered as a hazardous issue and can cause injuries such as bouncing a ball. Elasticity is a subject that requires hands-on practicality for learners to experience the effect of elastic objects. In this paper, we have investigated the interaction between learners and elastic objects naturally in a safe environment using GBL. During the interaction, the potentials of natural interaction in the learning process had been observed to investigate the exhibited gestures over the learning procedure. GBL was developed using Kinect technology to teach elasticity to children aged 7-12 years old in primary school. The procedure comprises of detecting the gesture of body and defining the meaning of gestures exhibited during the learning process. The quantitative approach was applied to constantly monitor the interaction between the student and the system. Based on our results, we found that Natural Interaction GBL (Ni-GBL) made students feel that the educational game is useful indirectly because they can learn how to play the game easily.
\end{abstract}

Keywords: $\quad$ Elasticity, Game-Based Learning (GBL), Kinect Technology, Natural Interaction.

\subsection{INTRODUCTION}

Natural Interaction or Interfaces Devices are devices that can capture body gesture, movement and sounds to allow interaction between user and computer[1]. Natural interaction exists when people interact with the system in the same way as they communicate with other people; this includes elements of expression, gestures, various intonations and movements. The term natural interaction is derived from Natural User Interface (NUI) and the use of the human body via verbal and non-verbal commands forming Human-Computer Interaction (HCI). This evolved from Graphical User Interface (GUI), the translation from commands to graphics to increase efficiency for the user. Kinect is an example of such a device [2], commonly used by gaming consoles such as Xbox 360. In 2012, Microsoft designed Kinect to be compatible with a PC, providing an opportunity for a wide variety of software and games developments [3]. Kinect detects the body gesture and communicates to the console or PC for transferring information and making a live game for the player. This has revolutionized interaction, as no conventional controller is required for interaction [4]. Kinect achieves controller-free gaming by using a depth sensor, a color camera, and a four-microphone array which can capture a full body 3D motion, facial and voice recognition [2,3]. Kinect can be one of the effective and interesting interaction devices to boost students' motivation in learning, provided that the learning material is suitable [5].

In this paper, a game to teach elastic objects to students aged 7 to 12 years old was developed and the level of their understanding, engagement and motivation were investigated. Although there is a huge number of educational games, elasticity educational games is limited. Elasticity is one of the important issues in physics and the number of elastic objects is limited in the real world, thus children are not familiar with elastic objects. In fact, this educational game and the unique idea behind it tries to clarify elastic objects for children using a playful attitude. In this research, we decided to use Kinect for simulating the environment and providing a safe and attractive environment. We chose Kinect because it provides a natural interaction for children to work with [5]. A common criticism of computer game is that they reduce the children's physical activity, which can lead to health problems such as obesity and lethargy. 
Kinect is one of the devices which can solve this issue [6]. Since the device is controlled by body movement, children have to physically move and exercise in order to play the game [5]. Moreover, children who play physical activity games burn more calories compared to the children who play non-activity video games [7]. In addition, Kinect is very interesting for children as it can detect their gesture. The main feature of Kinect is that children learn to increase their physical abilities without actually feeling like they are being forced. The main point of using Kinect is using game to teach children and they will learn without being aware of the actual training. We observed 30 different students who had been chosen randomly; then we analyzed the feedback of the cases and the results of the observation were proposed in the conclusion part.

In the next section, we present an overview of previous studies. Following the related work, we explain the rationale for the selection of natural interaction in our game and the definition of elasticity. Subsequently, the process of development and implementation of our research is presented and the methodology in our research is reported. In the seventh section describes data collection, analysis and discussion and finally, the conclusion is given in the last section.

\subsection{RELATED WORKS}

There are different interaction styles for game based learning such as physical, desktop, Virtual environments, XBOX360 and Microsoft Kinect environment. In the following studies, some of these interaction styles were considered for game based learning.

Falis (2005) considered the merits of desktop and physical environments for young children (4-6 years old) in a hazardous environment. The author compared the same content in both contexts in the purpose of comparing different learning environments. Falis provided a hazardous environment both in the desktop and a physical room. A pre and post-test for children was conducted, which were taken before and after the game respectively. The children answered the pre-test, then played the game in both environments and finally answered the post-test. The study concluded that physical environments have advantages over desktop environments. Although this study covered different measurements, the sample size was very small for indicating the advantages of physical environments over desktop environments [8].

Smith (2009) utilized Virtual reality (VR) to help children learn about fire hazards. The VR was applied for simulating dangerous situations to practice in real life and can also be considered as a tool to help children in learning and practicing escape techniques. A CAVE was used for immersing participants in a fire scene. In order to enhance the children's motivation to learn over prior VR fire-safety training methods, some game-like interface interaction techniques had been utilized for encouraging the children to find the virtual world. Rather than being passive viewers and in agreement with previous related studies, the children were controlled to navigate through the virtual environment, and to interact with virtual objects by a 6 DOF wand and a game pad. Students recognized the fire hazards at home with a partner and could practice escaping from the simulated fire in the virtual environment. To investigate the motivation improvement, an operator study was completed. Results show that students were more engaged by the new game-like learning environment and they initiated the experience as fun and intriguing. The results also show that students enthusiasm about the standard fire-safety information illustrated the potential of gamebased virtual environments improved [9].

Hedrick and Young [10] used a war game to increase the enhancement of the students in a surgical and clinical scenario. Based on their research, students performed poorly in the clinical performance. A game was developed which asked the students to verbalize management according to the report of the game. They received a reasonable result, especially for the senior medical students. The students' performance increased in the second and third round and above all, students were educated in a safe environment both for the patients and medical students. Educational game is one of the suitable solutions in some subjects, but for others, the impact is not evident in real practice. Wallace et al. [11] designed an educational game to teach road safety to children. Using the game developed, children were educated well and their knowledge about traffic behavior increased, but their study showed that a high proportion of their knowledge did not correspond with their actual traffic behavior.

Natural interaction game-based learning was used in a different purpose of education, for example, to educate disabled children. Sales, Cavalcante, Marques, and das Graças, (2011) developed an ALERV game which helped 
children with disability to learn how to read and write in Portuguese [12]. Voice recognition and Kinect Sensor were used in this research. The game was programmed in the C\# language and the XNA Game Studio 4.0 framework was used to connect the game with XBOX360 and body gestures were detected by Kinect. Voice recognition process was done by using the engine pt-BR (Brazilian Portuguese). The game recognized the sound produced by the children and checked whether it was correct in relation to the shape shown or not. Based on their research, they concluded that the process increased the children's motivation, activity and self-confidence.

In another work, educational games had been also used for disabled people to connect with larger societies. Solati provided an application to develop a gesture-based game for deaf/mute people [13]. In this project, Microsoft Kinect was used to scan the body movement of deaf/mute people, instead of their voice to control the game. In addition, this game assisted the disabled people to be more active physically. This game can be customized according to the problems of disabled people such as communication problem, independency and educational performance. Based on their observation, they received a positive feedback on how deaf/mute people communicate with others. As a result, the users were satisfied with the game and they enjoyed it.

Sucipto, A. et al implemented a game for gesture recognition on aquarium [14]. In this research, the researchers tried to provide a game using Kinect to detect the gesture limb movements of users to interact in an aquarium. They used $\mathrm{C}++$ open frameworks programming language and Linux operating system to develop the application. Gestures were recognized by utilizing open source libraries and OpenNI and NITE to process the image data from the Kinect. In this project, user interaction with the Interactive Aquarium was measured. Users were able to move objects in the game naturally using Kinect. There were some limitations in this project such as identifying the user from others who refused to recognize the calibration.

Boutsika (2014) considered the case of using Kinect as an educational tool for people with mobility problems, Boutsika proposed to extend the use for people with special needs, especially with autism. Children with autism are often competitive, and they want to lead and not to cooperate with other children. Boutsika tried to help the children to improve their behavior by making positive statements to each other and verifying their ability to help each other. He believed that one of the advantages of Kinect games is that they enable children to work in teams. This facilitates children to cooperate with each other and gradually develop their oral expression, coming to a point where they can give basic instructions to each other during the game. The students, upon the completion of the game, will gradually become familiar with the learning data and ideas, with the tools that will help them to learn and will increase the feeling of self-esteem, self-understanding, autonomy and independence. Additionally, through the game, children with autism will become familiar with various objects and concepts that will be slowly incorporated in their daily lives. At the same time, the games partially followed the pattern of repetitive and stereotyped behavior of children with autism, which will definitely make them react and not immediately reject this new way of education. Furthermore, the motion offered by Kinect is a solution to hyperactivity that characterizes people with autism in their daily activities. However, due to the specific case of their scenario, it was difficult to determine a method of assessment for the progress of the project. The assessment on the effectiveness of using Kinect to children with autism will be given through the children's personal experiences and efforts, and of course through the achievement or non-achievement of the expected results [15].

Difficulty in learning math is a common problem among children throughout the ages. The way of teaching and learning math have remained relatively the same in education. However, with the rise of portable devices; tablets and smart-phones, and motion-sensing controller such as the Microsoft Kinect, Nintendo WiiMote and the Playstation Move, people have shown a huge interest and motivation to develop new and interactive methods for teaching. Being inspired by this trend, Lee, Liu, \& Zhang (2012) had created an interactive, gesture-controlled arithmetic math game, Xdigit, using the Kinect to enhance and support the math learning experiences for children [16]. Children aged between 5 and 10 participated in this educational game. The player had the objective to combine numbers through arithmetic operations to match the target number before the timer runs out. The game came in various levels of difficulties to provide challenges and interesting mathematical opportunities by increasing the arithmetic complexity. The chosen theme for Xdigit was space, where the player encountered dangerous meteors on his or her journey to distant planets. These meteors were shown with the target number, which was pattern-generated and moved towards the player by a timer. In order to match the target number, one number was given for the arithmetic equation. The player's task was to select a number ( 1 to 9$)$ from the number wheel repository and combine that number with the given number using an arithmetic operator (plus, minus or 
multiply) with the Kinect gesture tracking. If the combined number matched the target number, the meteor will be destroyed and points will be given based on the remaining time. The technology behind Xdigit was based on the implementation of Microsoft Kinect's NUI skeletal tracking in a Unity3D game environment. They made a Kinect gesture system with trigger groups and skeleton information, and used Unity3D as the game engine combined with an open source animation/interpolation plugin system; iTween [16]. The results showed that Xdigit is a valuable platform to help children around the world with math learning difficulties. Xdigit does not only enhance and support math learning experiences, cognitive arithmetic and improve problem solving skills, but also creates a more fun environment for rich and meaningful experiences [16].

Synchronized online gymnastics may provide new possibilities for enhancing the physical and social well-being of people with restricted mobility. Cassola (2014) proposed a prototype platform for this - Online-Gym - which allowed users to interact using a Microsoft Kinect and participate in on-line gymnastics sessions. They presented the OnlineGym concept and the first iteration of the platform architecture that allowed interaction in OpenSimulator or Second Life virtual worlds with movements captured by a Kinect device. As outlined throughout this research, it was concluded that the Online-Gym project had a theoretical basis sustainable in the scope of a technology platform based on the Internet and had features relevant for users who were isolated, dispersed geographically or with reduced mobility, e.g., the elderly. It aimed to render them to be able to participate in group gymnastics activities. Remote assistants/facilitators may support these activities, rendering such a service available even in locations where a physical presence in a gym was not available or implied complex travel costs or logistics. The experimentation carried out within this project towards the architecture of such a system, showed that its implementation should take into account some requirements stemming from the existing systems integration, particularly in the synchronization of the movements and their impact on the network for other users, but also regarding the need to have a clear identification of the leader/monitor and custom controls for him/her. To conclude, they may state that the technological developments are currently ongoing in this area of integration between virtual 3D platforms and motion capture devices that rendered this project viable, albeit with a huge margin for improvement, meaning that there is the potential for the development and deployment of such services [17].

Another research on Kinect was done by González-Ortega, (2014). In this research, a real-time 3D computer visionaided system was provided and applied to the monitoring of psychomotor exercises. The system was intended for the assessment and evaluation of body scheme dysfunctions and left-right confusion. Monitoring was achieved through the human body joint tracking (head and hands) and the face and facial feature (eyes, nose, and ears) detection using the depth information provided by the Kinect device. Thus, the system had overcome the shortcomings of the 2D computer vision-based approaches and was robust to drastic changes in the working environment and illumination. The proposed system was capable of human limb monitoring; its analysis in relation to the psychomotor exercise was indicated to the user, and the storage of the result was provided for the realization of a set of exercises. The system was evaluated by 15 users, achieving a successful monitoring percentage of $96.28 \%$. This performance was suitable for the integration of the system in a multimodal cognitive rehabilitation platform [18].

Although current development toolkits were provided to support and identify the position of several joints of the human body and to process the movements of the body parts, they actually lacked a flexible and robust mechanism to perform high-level gesture recognition. Hence, developers are still left with the time-consuming and tedious task of recognizing gestures by explicitly defining a set of conditions on the joint positions and movements of the body parts [19]. Ibañez, (2014) presented a gesture-recognition tool to help developers to include gesture recognition in NUI applications. In particular, EasyGR enables developers to create, train and recognize gestures. For the first two activities, EasyGR brings a graphic interface that allows non-specialist users to capture gestures and train the recognition techniques. EasyGR supports the Dynamic Time Warping and Hidden Markov Models as recognition techniques. For the third, the developers have a template code for gesture recognition that they can include in their application, thus decreasing the development effort. Therefore, the main contribution of EasyGR is that it assists developers in the implementation of NUI applications. By encapsulating the gesture recognition, EasyGR reduces the complexity of managing the gesture data and the algorithms needed to build an NUI application. The results showed that EasyGR contributed to reducing the code size and complexity of gesture controlled applications. Furthermore, they had obtained correct recognition rates of over $99 \%$. In particular, it was observed that increasing the number of clusters used in HMM improved the gesture fidelity but made the gesture harder to imitate; thus the developer should find a balance between the gesture fidelity and the usability of the application. Nonetheless, EasyGR had some limitations. First, the gesture recognizer was unable to automatically adapt itself to 
the skills of the user. For instance, a developer creating a multilevel game would need a more restrictive recognizer every time the user completed each level. Although EasyGR did not have this feature, the developer can create different gestures to suit the user's skills. Second, they did not focus on new gesture-recognition techniques like Naïve-Bayes-Nearest-Neighbor and String Matching [19].

\subsection{NATURAL INTERACTION IN GAMES}

A natural interaction system can be successful while it is used individually. Natural interaction system with interesting media would be preferred by a majority of people. This type of systems can increase cognitive and cybernetic dynamics of the people; thus it can encourage people who are dealing with physical objects to give abstract and digital media as a second thought. It reduces the cognitive load, thus increases the amount of attention on the content [20].

Natural interaction mimics real world actions with a high level of fidelity[21]. Based on the history of machines, people have always adapted themselves to learn technological constraints to communicate with machines, while nowadays, it is possible to make machines adapted to humans' language in terms of sensation, presentation and narration. Natural interaction is one of these interaction paradigms which is defined in terms of some experiences such as people's communication through gestures, movement and expressions. In fact, interaction devices persuade humans to behave or interact dynamically. This means that the main key in natural interaction is the interaction of people with technology [22].

One of the benefits of the natural interaction system is the reduction of the cognitive load on the subjects. In addition, it engages people to interact and act naturally with each other and their environment; they do not need to use a device for controlling or learning any instructions. The interaction is visual, easy to work with, amazing, and interesting for everyone [20].

Kinect is one of the pioneers in natural interaction devices, especially in educational technology. Kinect, as a learning tool, simplifies and enhances education because it boosts the motivation and makes the learning process more enjoyable for students. In addition, it increases the physical activity of pupils during education and the process of their learning will be combined with some fun and interesting materials which can increase the level of their understanding [5].

In the following section, we explain how natural interaction can help students in an educational purpose to make it clear why we have chosen natural interaction games as our platform. One of the advantages of natural interaction devices compared to previous modalities or non-natural interaction devices such as mouse, keyboard and other devices is using "body organs" (like hands, face, head, voice etc.) which are always available because they are parts of the human body. In addition, they can be designed in a way to facilitate natural reactions and make users intuitive to focus on the task [23].

One way to understand the reason of using natural interaction in video games is to empirically evaluate the usability of such interaction techniques. Natural interaction techniques should be based on actions exhibited by players according to their expectation, age and their effort [24]. A manner towards understanding the effects of natural interaction is to compare natural interaction and non-natural interaction. According to McMahan, players have a better performance on natural interaction compared to the traditional games or non-natural techniques [21, 25]. In another research conducted by Höffler and Leutner, the effectiveness of multimedia games which involved human body movements is more than those which do not involve human movements [26]. As mentioned before, some natural interaction devices like Kinect were introduced to the educational system in order to help students to have a better education quality. Natural interaction device attracts more audience because it is more exciting to be played with; moreover, during the process of playing the game, it includes physical activity, movement and enjoyment which in return allow the pupils to be actively engaged in the game. As a result, pupils have higher motivation, engagement and fun [27]. Based on all of these advantages, we decided to present our multimedia game on a natural interaction device like Kinect. 


\subsection{ELASTICITY}

This section describes the elasticity formula which is applied to create the game. Elasticity is categorized into Young's modulus, Poisson's ratio, shear modulus, bulk modulus, compressibility, velocity of shear waves, and the velocity of compressional waves. The three primary categories are: Young's modulus, sheer modulus and bulk modulus [28]. In this research, we used Young's modulus as our refraction of elasticity object in our game because all the other elastic moduli can be derived from the Young's modulus and it is also known as material's stiffness which is the purpose of this project [29].

The theory of elastic modulus states objects inclination mathematically which is being able to transform when it goes under pressure as the opposite force is exerted on it. The elastic modulus of an object is described as the slope of its stress-strain curve in the elastic transformation region area [30,31] for example the elasticity of a rigid material will be higher than a soft object.

$\lambda \stackrel{\text { def }}{=} \frac{\text { Stress }}{\text { Strain }}$

For a better understanding, the relation of stress and strain is shown in Figure 1.

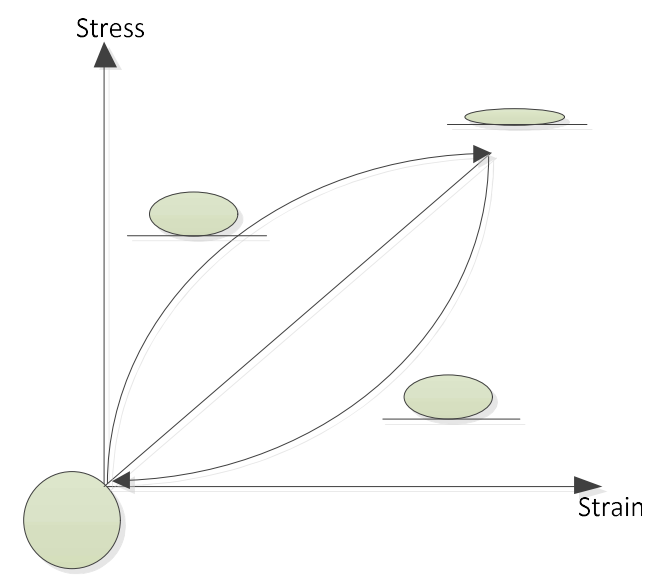

Fig 1: Relation of stress and strain in the elastic formula.

In this formula, lambda $(\lambda)$ refers to the elastic modulus and the stress identifies the resorting source which happens upon the transformation segmented by the region to the referred force. Moreover, stress clarifies the ratio of alteration and it is caused due to the stress to the primary situation of the object. Since strain is the kind of quantity with no dimension and stress is calculated in Pascal, the unit will be presented in Pascal [30, 31].

Describing how the stress and strain factors are to be calculated involving directions enables many kinds of elastic moduli to be specified.

Young's modulus (E) states tensile elasticity or in other words the inclination of an object, to disfigure along an axis when opposing the power that refers along that axis. It is often called the ratio of tensile stress to tensile strain. Sometimes, it is referred to as the elastic modulus. Young's modulus of elasticity formula was used in this paper, and the formula is shown below:

$$
\text { Elastic }=E=\frac{\text { Tensile Stress }}{\text { Tensile Strain }}
$$


$\mathrm{E}=\frac{\rho}{\varepsilon}=\frac{\mathrm{f} / \mathrm{A}_{0}}{\Delta \mathrm{L} / \mathrm{L}_{0}}=\frac{\mathrm{fL}_{0}}{\mathrm{~A}_{0} \Delta \mathrm{L}}=\frac{\mathrm{fL}_{0}}{\mathrm{~A}_{0}\left(\mathrm{~L}_{1}-\mathrm{L}_{0}\right)}$

Where $\mathrm{F}$ is the force applied on an object under tension. A0 is the original cross-section region through which the power is exerted. $\Delta \mathrm{L}$ shows how much the length of the object can vary and finally, L0 refers to the basic length of the object.

\subsection{DESIGN AND DEVELOPMENT}

The SDK provides the application programming interfaces (APIs) that are supported by the Microsoft Kinect drivers. In this research, we extended the shape game which was one of the SDK of the Microsoft Kinect toolkit to develop a game on the purpose of educating students in the range of 7-12 years old about elastic objects. Microsoft Kinect SDK and Visual Studio C\# 2010 Express were used to develop this game. The APIs control audio and video data streams, skeletal tracking of players who were moving within the Kinect sensor's field and different elastic and non-elastic objects were viewed in the Windows environment. The game was a natural interaction game which the player's body was gestured by Kinect and the user can control the game without using any tools (hands free control). In this study, the natural interaction design was based on the Kinect Microsoft toolkits and some of the existence of C\# classes and libraries. When the player stood in front of the Kinect, body gesture was detected by using an infrared projector and camera and a special microchip to track the movement of the objects and individuals in three dimensions. This $3 \mathrm{D}$ scanner system called Light Coding employed a variant of image-based 3D reconstruction.

A screenshot of the game is shown in Figure 2. When the game started, the body was detected by Kinect and a skeleton was drawn. The program was designed in a way which the player should only stand in front of the device and the distance of the player and Kinect should not be less than $150 \mathrm{~cm}$ or more than $300 \mathrm{~cm}$. Objects fell down from up with minimal gravity (0.025) and air friction (0.99). Half of the objects were created as having elasticity and the other half without elasticity. Shapes fell down with movements to the right or left to bop the wall, before being accessed by the children. When objects bopped to the walls, the elastic and non-elastic objects behaved as follows:

- Elastic objects were squeezed (around 2 seconds), bounced, the color changed to red and a squeeze sound was played.

- Non-elastic objects were not squeezed, bounced, stayed with the same color and there was no sound played for them.

The player should recognize the difference between elastic and non-elastic objects and remember them.

When the game was started, the point of the player was zero. As shown in Figure 2, the Star and pentagon objects in the left side were elastic. They showed elasticity with bopping to the wall and a sound of elastic was played. In addition, the color of the objects changed to red to make the elastic objects bolder for children. Players tried to squeeze and blast the objects. Blasting the elastic objects with different parts of the body generated different points. Table 1 shows the points of blasting elastic objects and non-elastic objects with different parts of the body.

Table 1: Points of blasting elastic objects and non-elastic objects.

\begin{tabular}{lll} 
& Elastic Objects & Non-Elastic Objects \\
\hline Head & +15 & -15 \\
\hline Hands & +5 & -5 \\
\hline Legs & +10 & -10 \\
\hline Walls & +1 & -1 \\
\hline
\end{tabular}

When the children blasted elastic objects, the game encouraged them with a "Yoho" sound and if they blasted the wrong object, there was a "No" sound to alarm them. Squeezing and touching without blasting (even for elastic or non-elastic objects) was not generated any points. 
The common mistake of the children was that they thought the elastic objects should be essentially spherical like a ball. Thus, in this project, there were seven different shapes such as: square, quarter, rectangle and star, for them to learn that the elastic object should not be necessarily a circle and it can be in any shape. Each shape had a different amount of elasticity according to its different cross section area through which the force was applied with different velocity and different area which touched the surface. Thus, based on these amounts of diversity, there were 26 different types of elasticity on these shapes.

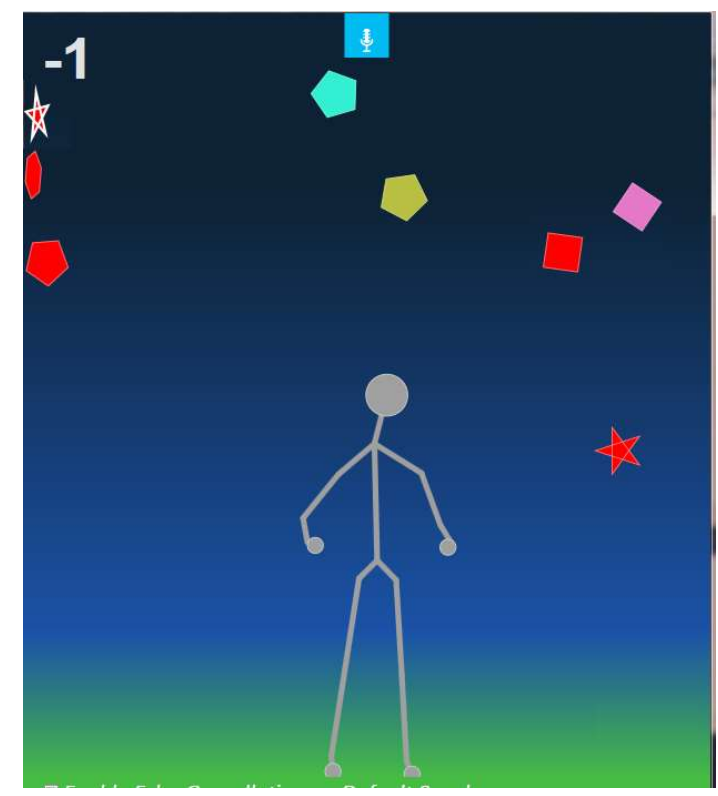

Fig 2: Application's screenshot.

\subsection{METHODOLOGY}

For data collection, a random public school in Malaysia was selected and 30 students aged between 7 to12 agreed to participate in the research. The students were chosen according to their ability and their educational levels were assessed. In addition, the students were selected from a mixture of well-behaved and misbehaved pupils and had different characteristics in terms of learning. The children were divided based on their ages in 2 different groups of 7 9 and 10-12 years old because the students with the similar age had almost the same understanding level, thus it will result in a more accurate and trustable outcome. Thus, during the rest of this study, group1 and group2 consisted of students aged of 7-9 and 10-12 years old respectively. In addition, the students were given 5 minutes of time to play the game, then the scores were recorded and a verbal test was conducted to evaluate them and all of the mentioned procedures were named as a cycle or round.

The metrics of comparison were quantitative. The quantitative analysis was based on the analysis of the game's score made by the student after each cycle and the percentage of students who passed the test. The game's score of the students were recorded after each cycle and the mean of scores was calculated. Regarding the test, it consisted of 8 questions which were administered verbally and the duration of the test took approximately 3 to 5 minutes. The questions were designed in a manner to cooperate with the game to teach the elasticity concept to the children. The questions are as follows:

"What is the difference of the red objects with the other objects?" Answer: any point to elasticity, stretch and etc..., 2 points.

"Did you find the objects which squeezed when they hit the wall or your body during the game?" Answer: yes 1 point.

"Is this sentence correct: 'Elastic objects such as ball, are squeezed when they hit the wall'? " Answer" yes 1 point. 
"What is the difference of a ball or squeezed objects in this game with a non-elastic object such as wood?" Answer: any point to: ball is elastic, stretch and etc..., 2 points.

"Why are some of the objects squeezed?" Answer: because they are elastic, 2 points.

"Is the ball squeezed the same as the squeezed objects in this game?" Answer: yes 1 point.

"How could you get the positive points or the sound of Yoho?" Blast the elastic, stretchiness, adaptable objects. 2 points.

"What is elasticity?" Answer: any point to stretchiness, adaptability or explanations such as 'the ability of an object or material to resume its normal shape after being stretched or compressed' are acceptable. 4 points.

The numbers of students who passed the test in each cycle were analyzed. Any point to the answers was accepted by the researcher for scoring the test. The last question was given 4 points because it was the main concept of learning in this game. With this scoring scheme, a completely correct test had a score of 15 .

Students were allowed to play the game in multiple rounds until they understood the difference between elastic and non-elastic objects. This meant that we gave them the freedom to play the game for more than one round until they could answer the given questions about the elasticity and earn at least the score of 10 from the test. Students who failed would be allowed to play the game once again and they entered another round. After each round, this was mentioned to the pupils who tried to find the answers of above-mentioned questions during the game. Then, the game was started for them again. The point of this game was teaching elasticity to children, thus, in each round, the students who understood the game and elasticity were eliminated. The game was continued until all of the students understood elasticity and all of them were eliminated from the game.

\subsection{RESULTS AND DISCUSSION}

To investigate the number and percentage of students who could understand elasticity at each round in the groups of 7-9 and 10-12 years old, Tables 2 and 3 were respectively prepared based on the results.

Table 2 shows the results of the understanding percentages of $0,13.33,46.66,86.66$ and $100 \%$ for group 1 at the first, second, third, fourth and fifth round respectively. Similarly, the understanding percentages for group2 were 0, 33.33, 86.66 and $100 \%$. First, it can be concluded that students with the higher ages presented a better performance compared with the younger students, indicating the direct connection between ages and the understanding concept, in a same topic.

It can be also noticed that the same behaviors can be observed by groups 1 and 2 in the first round. Most of them did not care about the educational purpose behind the game during the first round of playing. After the first round during the test, the meaning of elastic objects was explained for both groups to bring back their focus to the concept of elasticity. Most of the students in group 1 did not listen to the explanation, as they were excited to play the game and they regularly asked to play it again. In this round, only 2 students of group 1 could pass the test and were removed from the case study, but 13 of them could not pass the test and went to the second round to play the game on more time. Here, we referenced some of their answers which proved that they did not understand the meaning of elasticity. "Elastic objects are any objects", "Elastic objects could be squeezed while non-elastic objects could not", "Elasticity either can or cannot bounce", and some more meaningless definitions. Similarly, 2 students of group 2 understood the concept of elasticity in the second round. In contrast to the first group in which only 2 participants understood something about the game, for the second group, most of them said "objects are different and we think we get a positive or negative score based on the difference". As explained before, elastic objects will get a red color when bopped to the wall to make them more noteworthy. Most of the students in this range understood that there was something behind the red objects or red shapes which were the right shape to be squeezed and blasted, but they did not know about the elasticity that the game was trying to explain. In the third round, from the total of 13 students of group 1, 5 of them understood the game and 8 participants were not able to distinguish the elastic and non-elastic objects, while the majority of the students (11 students) in group2 could recognize the above-mentioned concept.

In the fourth round, in groups 1 and 2, there were the totals of 8 and 2 students respectively, who had to play the game again. As a result, the game was repeated for the fifth time for the students in group 1 with 2 remaining students, while all the students in group 2 passed the test in the fourth round. 
Finally, group 2 showed better results than the first group and the tests were finished after four rounds for this group, while there were five rounds for group1.

Table 2: The number and percentage of students who understood the game in the range of 7-9 years old.

\begin{tabular}{llllll} 
Round & First & Second & Third & Fourth & Fifth \\
\hline Understand person & 0 & 2 & 5 & 6 & $\%$ \\
\hline Understand Percentage & $\% 0$ & $\% 13.33$ & $\% 46.66$ & $\% 86.66$ & 00 \\
\hline Non-Understand person & 15 & 13 & 8 & 2 & $\% 0$ \\
\hline Non-Understand Percentage & $\% 100$ & $\% 86.67$ & $\% 53.34$ & $\% 13.34$ & $\% 0$
\end{tabular}

Table 3: The number and percentage of students who understood the game in the range of 10-12 years old.

\begin{tabular}{lllll} 
Round & First & Second & Third & Fourth \\
\hline Understand person & 0 & 2 & 11 & 2 \\
\hline Understand Percentage & $\% 0$ & $\% 33.33$ & $\% 86.66$ & $\% 100$ \\
\hline Non-Understand person & 15 & 13 & 2 & 0 \\
\hline Non-Understand Percentage & $\% 100$ & $\% 66.67$ & $\% 13.34$ & $\% 0$
\end{tabular}

Based on the observation, the students in both groups were very interested to play the game using Kinect. Games should be interesting and be able to engage the children. This will increase their motivation to learn using games. According to the results, this game had shown enough evidence that it was both engaging and interesting for pupils and it can boost their motivation for learning.

In this study, the means of the game's score that each group gained for each round were also analyzed. Figure 3 shows the means of the score for 7-9 year old students. It is evident that the students were weak in the first to third rounds, and the slope of score versus round showed an upward trend with a small slope. Their performance peaked in the fourth round with a sharp increase. They also played better in the fifth round, indicating that they understood the meaning of elasticity and they had become more comfortable with the game.

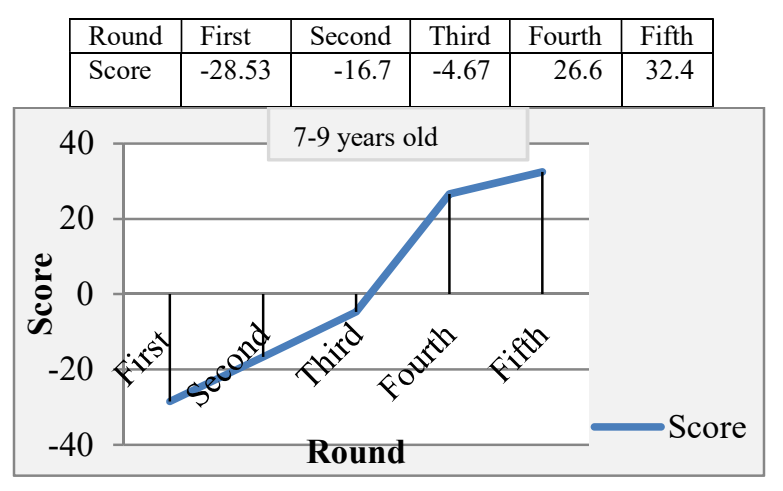

Fig 3: The mean score in each round for students in the range of 7-9 years old.

Figure 4 shows the students' score for the range of 10-12 years old in different rounds. First, the students could play the game better in this group as compared with group1. Then, the increase in score shifted to the third round, which 
was more significant than the results shown by group 1's results. It took up to four rounds for all the students to understand the concept of elasticity and the above-mentioned trend-shift, illustrating the effect of age on recognizing concepts. Also, as clearly shown in Figure 4, the majority of the students could get better scores than the younger students in different stages and this indicated more motivation to understand the game and play it in a proper way.

Overall, by comparing Figures 3 and 4, it was again clear that the older pupils (10-12 years old) could better understand elasticity one round before the pupils in the range of 7-9 years old. Around half of the students in the first group and more than half of the students in the second group understood the meaning of elasticity in the third round of playing the game. This might be due to them becoming excited using the Kinect; since this method was a new approach for them and being able to see the skeleton moves as they acted was intriguing to them. Kinect is a new game device which most of the students were not familiar with, but the enthusiasm of the students trying to play with it cannot be disregarded. Their excitement can trigger their motivation to play better without being aware of the actual learning procedure in the background.

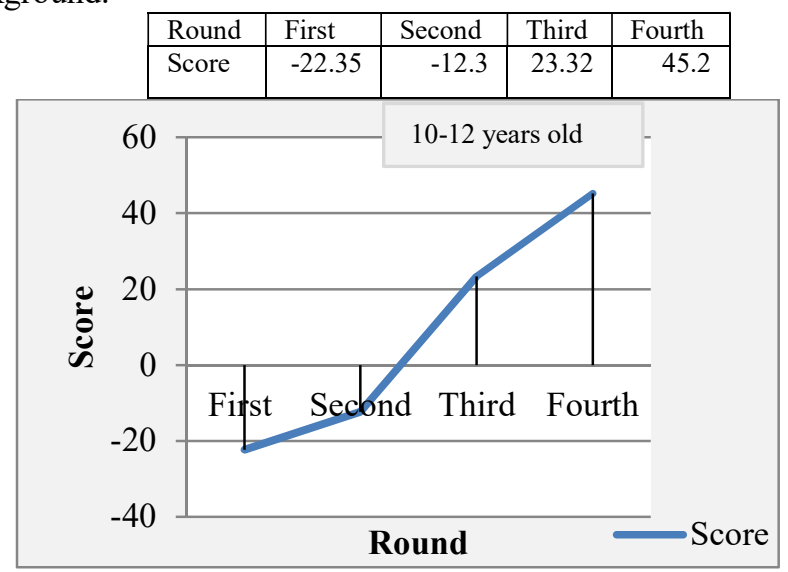

Fig 4: The mean score in each round for students in the range of 10-12 years old.

In the end of this section, our results were compared with the findings by Chang, Lachance et al. (2015), who had performed a close research to this study. Chang, et al. (2015) developed a Kinect game for students learning the law of orbit in physics. This study was conducted to verify whether the students' attitudes toward computer/video games will affect their perceptions toward the developed Kinect game or not, and whether their performance in the game will be influenced by the lack of prior knowledge of the law of orbits or not. The quantitative analysis of the results showed that the game can be used for everyone, despite the student's attitude towards computer and video games. Also, the game-play performance can be seen as a measure of the student's understanding of their prior knowledge. The use of arm and hand to play the game makes student perceive the game is easy to use and learn. Furthermore, this feature indirectly helped the student to perceive the usefulness of the game. Lastly, the play-withpeers feature not only had a positive correlation to the student's perceived usefulness towards the game, but can also made the game as a teaching assisted tool in the classroom for teachers. The results suggested that teachers may use the proposed natural user interface based game to assess their students' understanding of the correspondent knowledge in Physics. Moreover, for educational game developers and researchers, the results confirmed that natural user interface can make students feel that the educational game is useful indirectly due to they can learn how to play the game easily [32].

This study developed a Kinect game to learn elasticity, which is one of the physics laws, to the students. The quantitative results showed that since Kinect is one the newest devices which is currently used for educational purposes, students (especially the younger students) should be given the freedom to play until they become more familiar with it, because young students are excited about the game and it can reduce their focus for learning. However, natural user interface based game can be considered as one of the useful tools for education in the classroom. It can assist the teachers to educate hazardous and difficult issues easier because learners can actually be trained by simply acting in front of the Kinect without the acknowledgment of knowing that they are being educated. Finally, Kinect is an effective device for educational purposes because the results confirmed that natural user interface 
made the students felt that the educational game was indirectly useful indirectly due to they can learn how to play the game easily.

Table 4 shows the comparison with more description. It shows 20 students from grades 4 to 10 in pre-school and high school were chosen in their research. As mentioned before, in the present study 30 students aged 7-12 were chosen. As their game parameters, five factors were investigated: easy to learn, useful, liking, learning and confidence. We considered two of which in the present study including easy to learn and learning. Regarding the game time, it was unlimited in their research and students were free to play the game without considering the time. However as it is mentioned in this study, students after maximum of 5 minutes have stopped for quiz. Although our game was repeated five and four times for students aged 7-9 and 10-12, respectively, all students in these ages understood the concept of elasticity for maximum of 25 and 20 minutes respectively. Since Chang, et al. let students to play the game as long as they like, their method was time-consuming (around 50 minutes to 6 hours). The quantitative results in both researches were designed based on game score and test score. While Chang, et al.'s game resulted in $81.903 \%$ for easy to learn and useful factors and $67.54 \%$ for liking, learning and confidence factors for students, our results stood at $100 \%$ for easy to learn and learning factors in a reasonable time.

Table 4: A comparison between present study and a related work.

\begin{tabular}{|c|c|c|c|c|c|}
\hline & Students age & Game factors & Time & $\begin{array}{l}\text { The method of } \\
\text { quantity result } \\
\text { gathering }\end{array}$ & $\begin{array}{l}\text { The obtained } \\
\text { results for } \\
\text { game factors }\end{array}$ \\
\hline $\begin{array}{l}\text { (Chang, et } \\
\text { al., 2015) }\end{array}$ & $\begin{array}{l}20 \text { Pre-school and } \\
\text { high school students } \\
\text { (grade 4-10) }\end{array}$ & $\begin{array}{l}\text { Easy to Learn and } \\
\text { Useful }\end{array}$ & $\begin{array}{l}\text { Unlimited (Maximum time about } 6 \text { hours } \\
\text { Minimum time } 50 \text { minutes) }\end{array}$ & $\begin{array}{l}\text { Score based on the } \\
\text { game and questions }\end{array}$ & $81.903 \%$ \\
\hline $\begin{array}{l}\text { (Chang, et } \\
\text { al., 2015) }\end{array}$ & $\begin{array}{l}20 \text { Pre-school and } \\
\text { high school students } \\
\text { (grade } 4-10 \text { ) }\end{array}$ & $\begin{array}{l}\text { Liking, Learning and } \\
\text { Confidence }\end{array}$ & $\begin{array}{l}\text { Unlimited (Maximum time about } 6 \text { hours } \\
\text { Minimum time } 50 \text { minutes) }\end{array}$ & $\begin{array}{l}\text { Score based on the } \\
\text { game and questions }\end{array}$ & $67.54 \%$ \\
\hline $\begin{array}{l}\text { Present } \\
\text { Study }\end{array}$ & $\begin{array}{l}30 \text { students aged } 7- \\
9 \text { years old }\end{array}$ & $\begin{array}{l}\text { Easy to Learn and } \\
\text { Learning }\end{array}$ & $\begin{array}{l}\text { Limited to round and each round consist of } \\
5 \text { minutes. Game round repeated for } 5 \text { times } \\
\text { (Maximum } 25 \text { minutes). }\end{array}$ & $\begin{array}{l}\text { Score based on the } \\
\text { game and questions }\end{array}$ & $100 \%$ \\
\hline $\begin{array}{l}\text { Present } \\
\text { Study }\end{array}$ & $\begin{array}{l}30 \text { students aged } \\
10-12 \text { years old }\end{array}$ & $\begin{array}{l}\text { Easy to Learn and } \\
\text { Learning }\end{array}$ & $\begin{array}{l}\text { Limited to round and each round consist of } \\
5 \text { minutes. Game round repeated for } 4 \text { times } \\
\text { (Maximum } 20 \text { minutes). }\end{array}$ & $\begin{array}{l}\text { Score based on the } \\
\text { game and questions }\end{array}$ & $100 \%$ \\
\hline
\end{tabular}

\subsection{CONCLUSION}

As it is hazardous for children to learn about elastic objects in a real-life environment, this paper explored the possibility of educating children about elasticity of objects using Kinect. It can provide a safe environment for children to learn elasticity.

According to Hsu, Kinect is an efficient device for education [12], and since Kinect is a new device for education, the students should have the freedom to play until they become familiar with it. Student's excitement reduced their focus on the educational purposes of the game. Natural interaction game-based learning can be effective for learning since the learners can actually be trained by simply acting in front of the Kinect. Their motivation can be triggered by their movement in front of Kinect while interacting with the learning contents. Once the pupils are more comfortable with the Kinect, they can focus better on the learning material behind the game. Kinect is an effective device for educational purposes because the results confirmed that natural user interface can make the students feel that the educational game is useful indirectly due to they can learn how to play the game easily. Pupils' enthusiasm for interacting with Kinect cannot be disregarded because it promotes their motivation.

\section{ACKNOWLEDGEMENTS}

The authors gratefully acknowledge UMRG Grant RG085/11ICT, Faculty of Computer Science and Information Technology, University of Malaya, Malaysia for support to conduct this research work. 


\section{REFERENCES}

[1] A. Valli. "The design of natural interaction". Multimedia Tools and Applications, Vol. 38 No. 3,2008, pp. 295305.

[2] M.K. bin Mohd Sidik, M.S. bin Sunar, I. bin Ismail, M.K. bin Mokhtar, N. Jusoh. A study on natural interaction for human body motion using depth image data. Digital Media and Digital Content Management (DMDCM), 2011 Workshop on. IEEE2011. pp. 97-102.

[3] Z. Zhang. "Microsoft kinect sensor and its effect". MultiMedia, IEEE, Vol. 19 No. 2,2012, pp. 4-10.

[4] N. Villaroman, D. Rowe, B. Swan. Teaching natural user interaction using OpenNI and the Microsoft Kinect sensor. Proceedings of the 2011 conference on Information technology education. ACM2011. pp. 227-32.

[5] H.-m.J. Hsu. The Potential of Kinect as Interactive Educational Technology. 2nd International Conference on Education and Management Technology2011. pp. 334-8.

[6] S. Smallwood. "Energy expenditure of "Kinect"T" exergaming in schoolchildren". 2011.

[7] M.-E. Mathieu, L. Kakinami. "Active video games could be the solution to the increased energy intake reported with sedentary video games". The American journal of clinical nutrition, Vol. 94 No. 4,2011, pp. 1150-1.

[8] J.A. Fails, A. Druin, M.L. Guha, G. Chipman, S. Simms, W. Churaman. Child's play: a comparison of desktop and physical interactive environments. Proceedings of the 2005 conference on Interaction design and children. ACM2005. pp. 48-55.

[9] S. Smith, E. Ericson. "Using immersive game-based virtual reality to teach fire-safety skills to children". Virtual reality, Vol. 13 No. 2,2009, pp. 87-99.

[10] T.L. Hedrick, J.S. Young. "The use of "war games" to enhance high-risk clinical decision-making in students and residents". The American Journal of Surgery, Vol. 195 No. 6,2008, pp. 843-9.

[11] M.S. Zeedyk, L. Wallace, B. Carcary, K. Jones, K. Larter. "Children and road safety: Increasing knowledge does not improve behaviour". British Journal of Educational Psychology, Vol. 71 No. 4,2001, pp. 573-94.

[12] E.S.S.E.B. Sales, D.M. Cavalcante, L.B. Marques. "A Game for Teaching Children With disability in Reading and Writing in Portuguese using Voice Recognition and Kinect Sensor".

[13] F. Soltani, F. Eskandari, S. Golestan. Developing a gesture-based game for deaf/mute people Using microsoft kinect. Complex, Intelligent and Software Intensive Systems (CISIS), 2012 Sixth International Conference on. IEEE2012. pp. 491-5.

[14] A. Sucipto, A. Harsoyo, P.H. Rusmin. Implementation of gesture recognition on aquarium application. System Engineering and Technology (ICSET), 2012 International Conference on. IEEE2012. pp. 1-4.

[15] E. Boutsika. "Kinect in Education: A Proposal for Children with Autism". Procedia Computer Science, Vol. 27 2014, pp. 123-9.

[16] E. Lee, X. Liu, X. Zhang. "Xdigit: An arithmetic kinect game to enhance math learning experiences". Retrieved February, Vol. 14 2012, pp. 2013.

[17] F. Cassola, L. Morgado, F. de Carvalho, H. Paredes, B. Fonseca, P. Martins. "Online-Gym: a 3D virtual gymnasium using Kinect interaction". Procedia Technology, Vol. 13 2014, pp. 130-8. 
[18] D. González-Ortega, F. Díaz-Pernas, M. Martínez-Zarzuela, M. Antón-Rodríguez. "A Kinect-based system for cognitive rehabilitation exercises monitoring". Computer methods and programs in biomedicine, Vol. 113 No. 2,2014, pp. 620-31.

[19] R. Ibañez, Á. Soria, A. Teyseyre, M. Campo. "Easy gesture recognition for Kinect". Advances in Engineering Software, Vol. 76 2014, pp. 171-80.

[20] A. Valli. "Natural interaction". White Paper 2007.

[21] R.P. McMahan, A.J.D. Alon, S. Lazem, R.J. Beaton, D. Machaj, M. Schaefer, et al. Evaluating natural interaction techniques in video games. 3D User Interfaces (3DUI), 2010 IEEE Symposium on. IEEE2010. pp. $11-4$.

[22] A. Valli. "Natural interaction white paper". PhD Natural Interaction, iO Retrieved November, Vol. 12 2007, pp. 2008.

[23] N. Vidakis, M. Syntychakis, G. Triantafyllidis, D. Akoumianakis. Multimodal natural user interaction for multiple applications: The gesture-Voice example. Telecommunications and Multimedia (TEMU), 2012 International Conference on. IEEE2012. pp. 208-13.

[24] L. Loke, A.T. Larssen, T. Robertson, J. Edwards. "Understanding movement for interaction design: frameworks and approaches". Personal and Ubiquitous Computing, Vol. 11 No. 8,2007, pp. 691-701.

[25] R.P. McMahan, D. Gorton, J. Gresock, W. McConnell, D.A. Bowman. Separating the effects of level of immersion and 3D interaction techniques. Proceedings of the ACM symposium on Virtual reality software and technology. ACM2006. pp. 108-11.

[26] T.N. Höffler, D. Leutner. "Instructional animation versus static pictures: A meta-analysis". Learning and instruction, Vol. 17 No. 6,2007, pp. 722-38.

[27] C.Y. Chang, Y.T. Chien, C.Y. Chiang, M.C. Lin, H.C. Lai. "Embodying gesture-based multimedia to improve learning". British Journal of Educational Technology, Vol. 44 No. 1,2013, pp. E5-E9.

[28] G. Simmons. Single crystal elastic constants and calculated aggregate properties. DTIC Document1965.

[29] R.W. Westreich, H.-W. Courtland, P. Nasser, K. Jepsen, W. Lawson. "Defining nasal cartilage elasticity biomechanical testing of the tripod theory based on a cantilevered model". Archives of Facial Plastic Surgery, Vol. 9 No. 4,2007, pp. 264-70.

[30] D.R. Askeland, P.P. Fulay, W.J. Wright. The science and engineering of materials. Thomson Engineering2011.

[31] R.M. Jones. Mechanics of composite materials. Taylor \& Francis London1975.

[32] M. Chang, D. Lachance, F. Lin, F. Al-Shamali, N.-S. Chen. "Enhancing Orbital Physics Learning Performance through a Hands-on Kinect Game". Egitim ve Bilim, Vol. 40 No. 180,2015. 